\title{
UCRL-PROC-215251
}

LAW RENCE LIVERMORE N A T IO N A L LABORATORY

\section{Polarization Smoothing on the National Ignition Facility}

S. N. Dixit, D. Munro, J. R.. Murray, M. Nostrand, P. J. Wegner, D. Froula, C. A. Haynam, B. J. MacGowan

September 10, 2005

Inertial Fusion Science and Applications Biarritz, France September 4, 2005 through September 9, 2005 
This document was prepared as an account of work sponsored by an agency of the United States government. Neither the United States government nor Lawrence Livermore National Security, LLC, nor any of their employees makes any warranty, expressed or implied, or assumes any legal liability or responsibility for the accuracy, completeness, or usefulness of any information, apparatus, product, or process disclosed, or represents that its use would not infringe privately owned rights. Reference herein to any specific commercial product, process, or service by trade name, trademark, manufacturer, or otherwise does not necessarily constitute or imply its endorsement, recommendation, or favoring by the United States government or Lawrence Livermore National Security, LLC. The views and opinions of authors expressed herein do not necessarily state or reflect those of the United States government or Lawrence Livermore National Security, LLC, and shall not be used for advertising or product endorsement purposes. 


\title{
Polarization Smoothing on the National Ignition Facility
}

\author{
S. N. Dixit, D. Munro, J. R. Murray, M. Nostrand, P. J. Wegner, D. Froula, \\ C. A. Haynam and B. J. MacGowan \\ Lawrence Livermore National Laboratory, L-462, P. O. Box 808, Livermore, CA 94551
}

\begin{abstract}
We have recently implemented polarization smoothing (PS) on one quad of the NIF laser. Specially cut KDP and DKDP crystals at $420 \times 420 \mathrm{~mm}$ sizes were used to scramble the incident $351 \mathrm{~nm}$ laser polarization over the beam aperture. The intensity contrast deduced from the measured focal spot images for one of the NIF beams is in very good agreement with the expected contrast. KDP and DKDP crystals are known to produce considerable amount of transverse stimulated Raman scattering (SRS) when irradiated with large beams at $\sim 1-2 \mathrm{GW} / \mathrm{cm}^{2}$. In order to measure the transverse SRS, we attached optical fibers on the side of one of the PS crystals. The KDP PS crystal showed $>1 \mathrm{~J} / \mathrm{cm}^{2}$ side scattered SRS at irradiances of $1.2 \mathrm{GW} / \mathrm{cm}^{2}$. The DKDP (70\% deuteration level) PS crystal showed significantly less SRS. Detailed analysis of the SRS scattering in the PS crystal is in progress.
\end{abstract}

\section{INTRODUCTION}

High power fusion lasers such as the National Ignition Facility laser produce non-diffraction limited focal spots due to static wavefront aberrations from imperfect optic finishing and dynamic wavefront errors due to pump non-uniformities and non-linear effects. The focal spots of such beams contain many high intensity hot-spots that could seed laser plasma instabilities (LPI) such as filamentation, stimulated Raman and Brillouin scattering etc. at the target. It is now widely accepted that spatial and temporal beam conditioning is required in order to control and possibly suppress LPI effects in fusion plasmas. Spatial beam conditioning involves the use of continuous phase plates to broaden the focal spot to produce a desired spot envelope (Supergaussian, elliptical etc). The speckle pattern overlaying this envelope is smoothed using techniques such as smoothing by spectral dispersion (SSD) and polarization smoothing (PS). The effectiveness of this smoothing is characterized by the reduction of the intensity contrast in the smoothed focal spot. Statistics of speckle averaging indicates that an intensity addition of $\mathrm{N}$ uncorrelated speckle patterns reduces the speckle contrast by a factor of $1 / \sqrt{ } \mathrm{N}$.

Polarization smoothing (PS) is a process wherein two independent speckle patterns corresponding to two orthogonal polarizations are overlapped to achieve smoother focal spot intensity profiles. The main advantage of PS is that it is effective instantaneously and does not require any time averaging. The limitation of PS is that the maximum reduction in the intensity contrast is $1 / \sqrt{ } 2$ because only two orthogonal polarization directions are available. The instantaneous nature of PS is believed to help suppress filamentation and other LPI effects.

\section{IMPLEMENTATION OF PS ON NIF}

The effectiveness of PS depends on being able to generate two uncorrelated speckle patterns of nearly equal average intensity with orthogonal polarizations and overlapping them at the target plane. One implementation of this using a KDP birefringent wedge has been done on the Omega Laser facility at the Laboratory for Laser Energetics at the University of Rochester. Hardware constraints on NIF necessitated placing the PS crystal in the converging part of the NIF beam after the focus lens. (see fig 1) The design method for the PS crystals in a converging beam is described in ref [1]. We considered $420 \times 420 \times 10 \mathrm{~mm}$ sized KDP and the DKDP (70\% deuteration level) crystals for PS. The cut angles for these crystals in the boule were determined by requiring that the focal spots produced for the 'o' and the 'e' components be offset 
by $30 \mu \mathrm{m}$ from each other. This is illustrated in fig 2 . These crystals were cleaned and anti-reflection coated for $351 \mathrm{~nm}$ before being used in the laser.

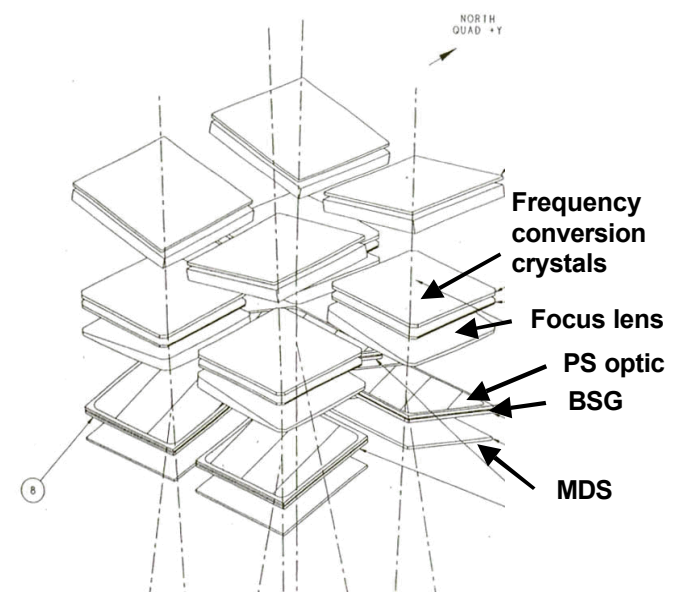

Figure 1. Final optics configuration for the NIF Early Light quad. The beams are propagating towards the bottom of the page.

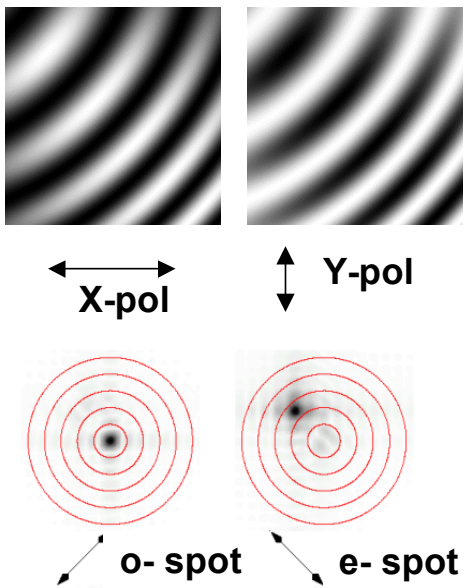

Figure 2. (Top) Expected near-field intensity pattern in the two orthogonal polarization directions shown. (Bottom) Expected unaberrated focal spots for the 'o' and the 'e' polarizations. Each circle corresponds to a $15 \mu \mathrm{m}$ increase in radius.
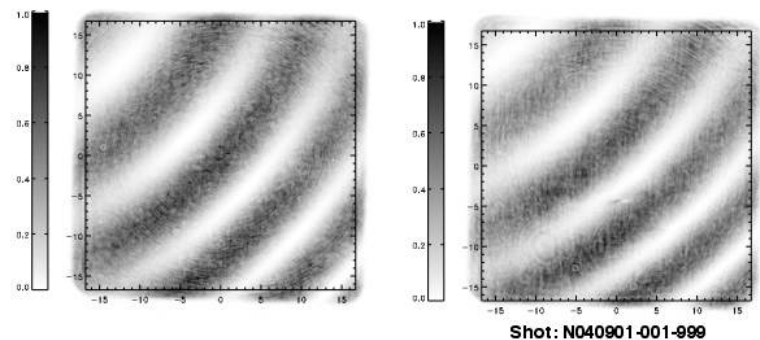

Figure 3. Expected (left)and measured (right) polarized near-field intensity pattern.

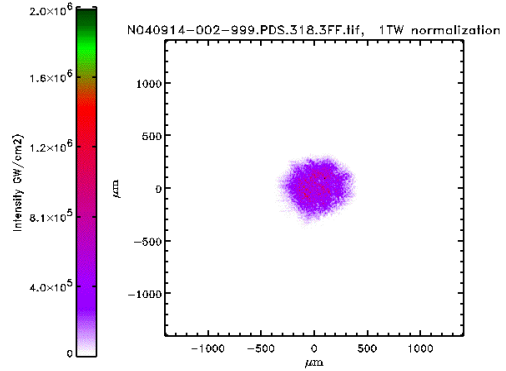

Figure 4. Measured $3 \omega$ focal spot with a CPP and a PS crystal in the beam.

\section{RESULIS}

\subsection{PS performance}

The optical characterization of PS was done by diverting one NIF beam line into the precision diagnostic station (see ref 2 for details). The polarized near-field intensity and the focal spot were measured to characterize PS. Figure 3 compares the measured and the expected near-field intensity profiles in a single polarization after the $351 \mathrm{~nm}$ laser beam has passed through the PS plate. The excellent agreement between the two profiles confirms that the PS plate is performing as designed. Figure 4 shows the focal spot intensity measured with a full aperture continuous phase plate and the PS crystals in the beam path. The measured intensity contrast over the central $200 \mu \mathrm{m}$ region is 0.35 which is less than the expected $0.70(=$ $1 / \sqrt{ } 2$ ) due to PS. This is understood as follows: our far-field camera has a lower resolution which reduces the speckle contrast to 0.5 before PS and PS further reduces this by $1 / \sqrt{2}$ leading to an effective contrast of $0.35(=0.7 * 0.5)$. This combined with the near-field polarized intensity profile confirms that the PS on NIF is working as designed.

For the target experimental campaigns using the NEL quad, PS was implemented on all four beams using DKDP crystals. The results of these campaigns are discussed elsewhere in these proceedings $[3,4]$

\subsection{Transverse SRS in PS crystals}


Four UV optical fibers were attached to the PS crystal frame to measure the amount of side scattered SRS light (fig 5). The measured SRS fluence is plotted in figure 6 for KDP and DKDP PS crystals. The fit to this data using a 1.5 pass SRS gain formula (corresponding to a $3 \mathrm{~ns}$ pulse) implies that the gain coefficients are comparable to the non-PS crystal gain coefficients measured elsewhere. A detailed investigation of the SRS scattering in a PS crystal is in progress. These high SRS scattering levels in the PS crystals increase the damage risk for the optic and the surrounding hardware during the laser operation at ignition energies.

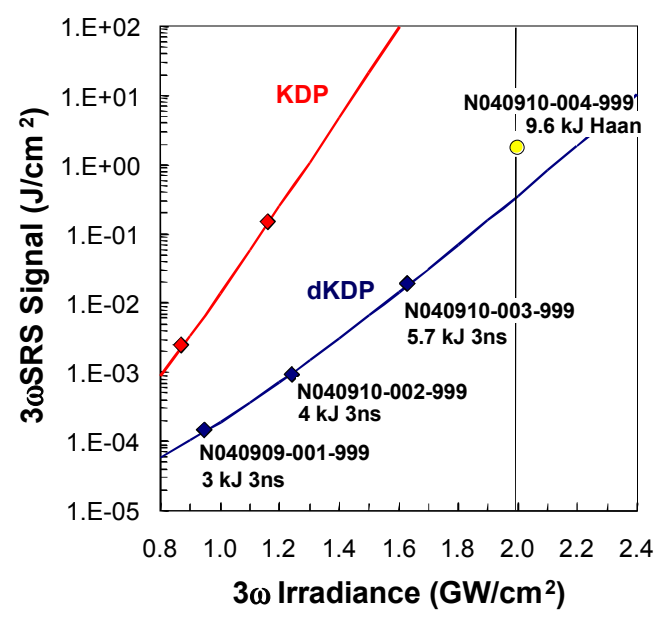

Figure 5. Variation of the transverse SRS signal in the PS crystals as a function of the incident irradiance. The deduced gain coefficients for $\mathrm{KDP}$ and DHDP are 0.3 and0.. $18 \mathrm{~cm} / \mathrm{GW}$.

\section{Checkerboard PS on NIF}

In order to minimize the transverse SRS scattering in the PS crystals and reduce the damage threat to the PS crystals, we are currently investigating a checkerboard PS scheme for NIF. Here the polarization of the half of the beams is rotated by 90 degrees relative to the other half using full aperture half wave plates. PS is achieved by overlapping the focal spots of the rotated and the unrotated beams in the target plane. The advantages of this approach are: the polarization rotation can be performed in the $1.053 \mu \mathrm{m}$ section of the beamline, we need only half as many polarization rotation (PR) crystals and there would be negligible SRS in these PR plates in the $1.053 \mu \mathrm{m}$ section. The challenges in implementing this scheme on NIF are the need to rotate the frequency conversion crystals on half the beams to match the rotated laser polarization and the limitation that the PS is effective only when the multiple beams are overlapping. Initial plasma simulations seem to indicate that this is not a severe limitation.

\section{Summary}

Using specially cut KDP and DKDP crystals, we demonstrated PS on one quad of the NIF laser. The measured PS performance is as expected. The PS crystals produced considerable amount of transverse SRS light which might limit the maximum available power during operation. To overcome the limitations of the intrabeam PS, we are examining rotating the polarizations of two beams in the NIF quad and overlapping the rotated and the unrotated beam speckle patterns in the target plane

\section{Acknowledgement}

Work performed under the auspices of the U.S. Department of Energy by University of California, Lawrence Livermore National Laboratory under Contract No. W-7405-ENG-48.

\section{References}

[1] D. H. Munro et. al. "Polarization smoothing in a convergent beam", APPl. Opt. 43 (2004) 6639-6647.

[2] See the paper by C.. Haynam in these proceedings.

[3] See the paper by D.. Hinkel in tehse proceedings

[4] See the poster by M. Schneider in these proceedings. 